\title{
DYNAMICAL BEHAVIOR OF FUZZY LOGIC BASED VELOCITY CONTROL AUTOPILOT WITH RESPECT TO CHANGES IN LINGUISTIC VARIABLES MEMBERSHIP FUNCTIONS SHAPE
}

\author{
Petya KOPRINKOVA and Valentine PENEV
}

\section{Introduction}

Recently, fuzzy control has become one of the most attractive areas of fuzzy set theory applications. ${ }^{3,10}$ As it provides an effective way to approximate inexact nature of human thinking, this theory is an appropriate tool for converting linguistically expressed expert's knowledge about a given process control into mathematically defined control strategy. This is made by means of the so called linguistic values, which are fuzzy sets over given universe of discourse of corresponding linguistic variable. The membership functions, which describe this linguistic values, as a rule, are defined by some bell-shaped function. Several nonlinear definitions of this functions are known, but most researchers prefer to use simple linear triangular or trapezoidal functions. Usually in fuzzy controller synthesis membership functions shape is defined in advance and then only their position in the universe of discourse is tuned.

It is shown that the bell shaped membership functions appear to be more appropriate for some cases of fuzzy control systems because the shape of the membership functions influences the shape of the fuzzy controller actions surface and, hence, the dynamics of the system under control, as well as its stability.

In the present paper the investigation of the influence of nonlinearities in membership functions shape on fuzzy logic based velocity control autopilot dynamics is investigated. 


\section{Problem Statement}

The concept of "velocity control" ${ }^{4}$ is associated with manual control systems where the operator tracks both the missile and the target and attempts to bring target and missile into alignment by issuing commands to the missile, usually by means of a specially designed thumb controller and a command link to the missile. If no autopilot is used then a constant demand - either up-down or left-right - will result in the missile developing a steady lateral acceleration.

Consider the action necessary by the operator to make a correction in position in the yaw plane. To put in a left correction a good operator will put over his controller to the left until half the "error" is eliminated and will then reverse his controller. Ideally, transients in the missile should be so short that the operator is not really aware of their presence. A great deal of anticipation and training is required to develop such a skill; the operator is using his own intelligence to supply the necessary amount of phase advance.

Consider a missile carrying a position gyro which feeds back a voltage proportional to yaw angle. The situation now is that the net signal into the rudder is the difference between the command and the achieved angular position of the missile. When the missile is pointing in the desired direction the rudder will automatically be returned to the central position and the missile will continue to fly straight in this direction. Such a system of controlling the trajectory of a missile is called "velocity control." The opinion is widely held that the training of operators is much easier with velocity control than with acceleration control. Another advantage of a gyro in the loop is that the missile will automatically react to a wind gust, thrust misalignment or disturbance due to a rigging error. With antitank missiles there is always the danger of hitting the ground. When a position gyro is used in the pitch plane the missile is usually launched at a set elevation with the gyro uncaged at an angle to the missile framework so that it will fly in a horizontal plane with a small permanent incidence to overcome gravity. Without the gyro, gravity compensation still has to be obtained. Any error in biasing the elevator servos results in the missile accelerating upwards or downwards and this calls for more skill from the operator if he is to bring the missile back on course.

A velocity control system is suitable for systems designed to hit stationary or slow moving targets where small adjustments only are required by the operator. LOS systems designed to hit fast crossing targets require the missile to execute a curved trajectory and a directional autopilot would merely hinder the operator in trying to keep the missile in the LOS.

A block diagram of a velocity control autopilot ${ }^{4}$ is shown in Figure 1. 


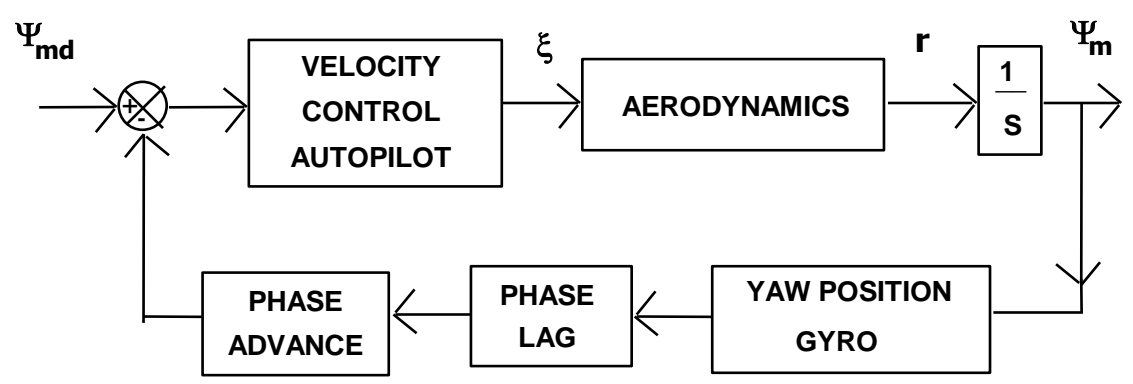

Figure 1. A velocity control autopilot.

Here $r$ is rudder, $\xi$ is body rate, $\Psi_{m}$ is body direction and $\Psi_{m d}$ is the desired body direction.

\section{Fuzzy Controller and Membership Functions Description}

Fuzzy controllers are described by means of some linguistic control rules set. One of the most popular type of rules is used in the present investigation:

$$
\text { IF } x_{1} \text { is } L_{j} \text { and } x_{2} \text { is } L_{k} \text { and ... } x_{p} \text { is } L_{q} \quad \text { THEN control action is } u_{i}
$$

where $i$ is number of control rule, $i=1 \div N, N$ is number of rules, $L_{j}, L_{k}, \ldots, L_{q}$ are linguistic values of the controllers' input variables $x_{l}, \ldots, x_{p}$ respectively and $u_{i}$ is crisp control action for $i$-th rule.

The crisp value $u$ is a result of composition of all fuzzy rules with some compositional rule of inference and defuzzification. Usually, max-min composition and centre of gravity defuzzification are used. In this case the crisp value is obtained as follows:

$$
u=\frac{\sum_{i=1}^{N} w_{i} u_{i}}{\sum_{i=1}^{N} w_{i}}, \text { where } w_{r}=\min _{i=1}^{p} L_{k}\left(x_{i}\right), r=1 \div N
$$

Here $\mu$ denotes the membership grade of corresponding inputs of controller $\left(x_{1}, \ldots, x_{p}\right)$ with respect to given linguistic values $L_{j}, \ldots, L_{q}$ and $w_{i}$ is the degree in which $i$-th rule influences controller output.

The following parametrized functions for increasing and decreasing parts of membership functions are proposed ${ }^{2}$ : 


$$
\begin{aligned}
& \mu_{I}(x)=\frac{(1-\beta)^{\alpha-1}(x-a)^{\alpha}}{(1-\beta)^{\alpha-1}(x-a)^{\alpha}+\beta^{\alpha-1}(c-x)^{\alpha}}, x \in[a, c] \\
& \mu_{D}(x)=\frac{(1-\beta)^{\alpha-1}(b-x)^{\alpha}}{(1-\beta)^{\alpha-1}(b-x)^{\alpha}+\beta^{\alpha-1}(x-c)^{\alpha}}, x \in[c, b]
\end{aligned}
$$

where $\alpha$ is parameter defining the function slope and $\beta$ is the inflection point of the curve. Parameters $a, b$ and $c$ define the support (definition interval boundaries) of the function. The indexes $I$ and $D$ denote the increasing and decreasing function respectively. The influence of these two parameters on the shape of the membership function can be seen on Figure 2.

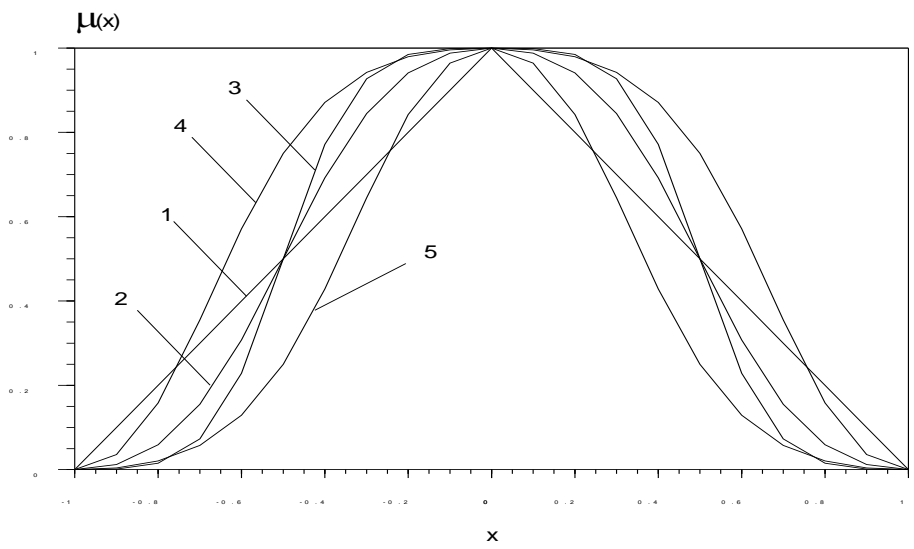

Figure 2. Influence of $\alpha$ and $\beta$ parameters on the shape of membership function. curve 1 - $\alpha=1$; curve 2 - $\alpha=2, \beta=0.5$; curve 3 - $\alpha=3, \beta=0.5$; curve 4 - $\alpha=2, \beta=0.25$; curve 5 - $\alpha=2, \beta=0.75$.

\section{Simulation Results and Discussion}

The object under control is the missile (Figure 1) described by the aerodynamics with the following transfer function:

$$
\frac{r}{\xi}=\frac{\left(T_{i} s+1\right) k_{a}}{s^{2} / \omega_{n w}^{2}+s 2 \mu_{w} / \omega_{n w}+1}
$$


The parameter values are as follows:

$$
T_{i}=0.043 \quad k_{a}=20 \quad \omega_{n w}^{2}=\frac{1}{0.01} \quad 2 \mu_{w}=0.3 \omega_{n w}
$$

The controlled variable is body direction $\Psi_{m}$. It has to follow the desired body direction $\Psi_{m d}$. The velocity control autopilot incorporates the operator's skills in the form of fuzzy control rules, described in the previous section.

The control action $\xi(t)=u(t)$ is calculated according to the fuzzy rules table (Table 1). The fuzzy controller inputs are the error $e(t)$ and its change $d e(t)\left(x_{1}=e(t), x_{2}=d e(t)\right)$, i.e.:

$$
e(t)=\Psi_{m d}(t)-\Psi_{m}(t) \quad d e(t)=e(t+\Delta t)-e(t)
$$

Here $\Delta t=0.001$ is the integration step. The supports of the corresponding fuzzy values of the two input linguistic variables $(e$ and $d e$ ) are given in the Table 2 and Table 3 respectively.

\begin{tabular}{|c|c|c|c|}
\hline \multirow{2}{*}{$\boldsymbol{d} e(t)$} & \multicolumn{3}{|c|}{$\boldsymbol{e}(\boldsymbol{t})$} \\
\cline { 2 - 4 } & $\boldsymbol{L}_{\boldsymbol{1}}$ & $\boldsymbol{L}_{\mathbf{2}}$ & $\boldsymbol{L}_{\mathbf{3}}$ \\
\hline \multirow{2}{*}{$\boldsymbol{L}_{\boldsymbol{1}}$} & 0.0 & +0.005 & +0.01 \\
\hline $\boldsymbol{L}_{\mathbf{2}}$ & -0.005 & 0.0 & +0.005 \\
\hline $\boldsymbol{L}_{\mathbf{3}}$ & -0.01 & -0.005 & 0.0 \\
\hline
\end{tabular}

Table 1. Fuzzy controller rules.

\begin{tabular}{|c|c|c|c|}
\hline $\boldsymbol{e}(\boldsymbol{t})$ & $\boldsymbol{a}$ & $\boldsymbol{b}$ & $\boldsymbol{c}$ \\
\hline $\boldsymbol{L}_{\boldsymbol{1}}$ & -0.05 & 0.0 & -0.05 \\
\hline $\boldsymbol{L}_{\boldsymbol{2}}$ & -0.05 & +0.05 & 0.0 \\
\hline $\boldsymbol{L}_{3}$ & 0.0 & +0.05 & +0.05 \\
\hline
\end{tabular}

Table 2. Membership functions supports for the error $e$. 


\begin{tabular}{|c|c|c|c|}
\hline $\boldsymbol{d e}(\boldsymbol{t})$ & $\boldsymbol{a}$ & $\boldsymbol{b}$ & $\boldsymbol{c}$ \\
\hline $\boldsymbol{L}_{\boldsymbol{1}}$ & -0.0005 & 0.0 & -0.0005 \\
\hline $\boldsymbol{L}_{\boldsymbol{2}}$ & -0.0005 & +0.0005 & 0.0 \\
\hline $\boldsymbol{L}_{3}$ & & & +0.0005 \\
\hline
\end{tabular}

Table 3. Membership functions supports for the change of error $d e$.

Simulation results with different values of the parameters $\alpha$ and $\beta$ of fuzzy controller membership functions are shown on the Figure 3, 4 and 5.

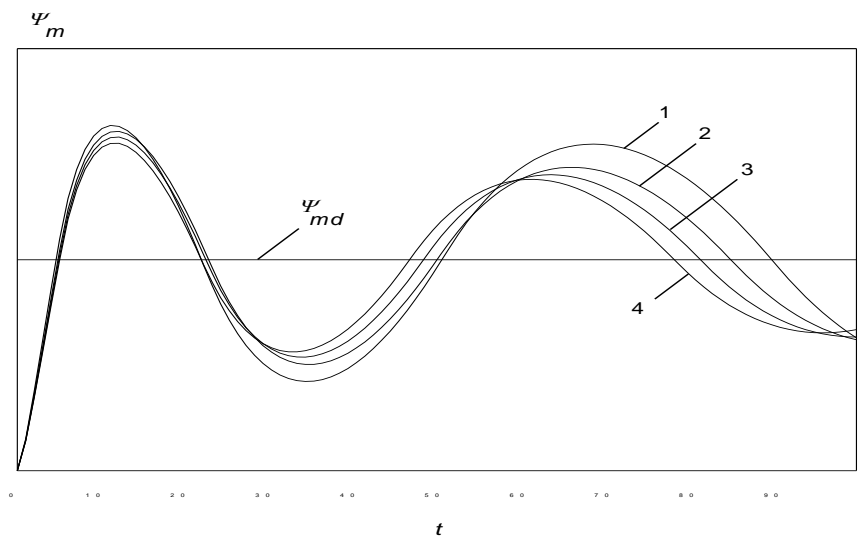

Figure 3. Simulation results with $\beta=0.25$ and different values of $\alpha$ (time duration 1s). curve $1-\alpha=1$; curve 2 - $\alpha=2$; curve 3 - $\alpha=3$; curve 4 - $\alpha=4$.

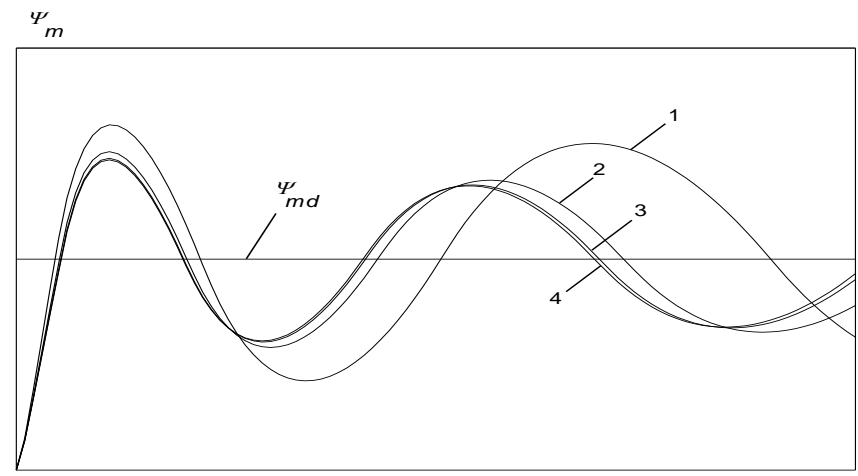

Figure 4. Simulation results with $\beta=0.5$ and different values of $\alpha$ (time duration $1 \mathrm{~s}$ ). curve $1-\alpha=1$; curve $2-\alpha=2$; curve $3-\alpha=3$; curve $4-\alpha=4$. 


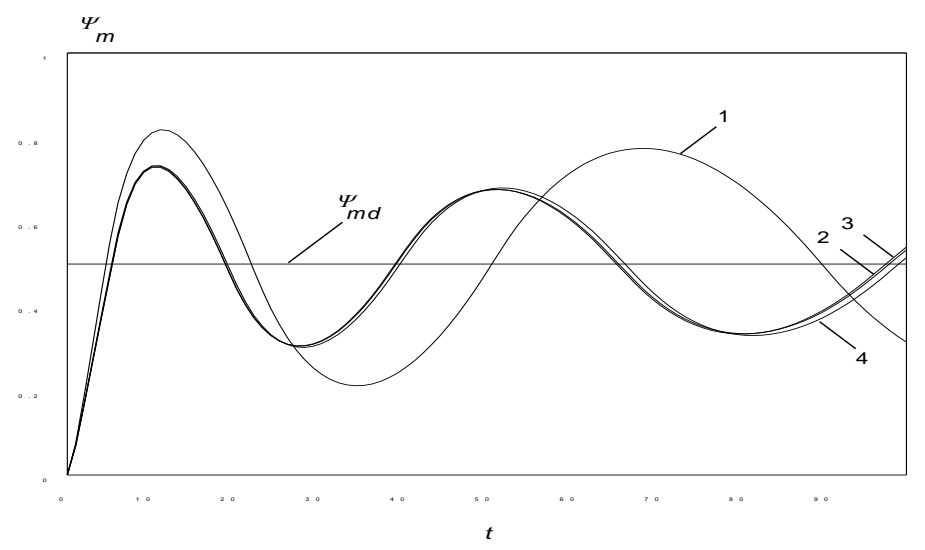

Figure 5. Simulation results with $\beta=0.75$ and different values of $\alpha$ (time duration $1 \mathrm{~s}$ ).. curve 1 - $\alpha=1$; curve 2 - $\alpha=2$; curve $3-\alpha=3$; curve $4-\alpha=4$.

As can be seen from the above figures, fuzzy control autopilot with bell-shaped membership functions decreases the amplitude and period of fluctuations around the set point in comparison with the triangular-shaped membership functions in all the cases of parameters $\alpha$ and $\beta$. With the increase of parameter $\beta$ (inflection point) the period of fluctuations decreases. With increasing of parameter $\alpha$ in the cases $\beta=0.25$ and $\beta=0.5$ (figures 3 and 4 ) the period and amplitude of fluctuations decrease, but for $\beta=0.75$ there is no significant difference in simulation curves (Figure 5).

\section{Conclusions}

In the present paper the influence of the two parameters defining the linguistic variables membership functions shape on the dynamical behaviour of fuzzy control autopilot for missile is investigated. The simulation results led to the conclusion that the bell-shaped membership functions give better results in comparison with the triangular once.

\section{Acknowledgement}

The work on this paper was supported by the Bulgarian Academy of Sciences, project "Development of Moving Platform simulator".

\section{References}

1. Baglio, S., Fortuna, L., Graciani, S. and Muscato, G., "Membership Function Shape and the Dynamical Behaviour of a Fuzzy System," First European Congress on Fuzzy and Intelligent Technologies EUFIT'93 (Sept. 7-10, 1993, Aachen, Germany), 645-650.

2. Dombi, J., "Membership Function as an Evaluation," Fuzzy Sets and Systems 75 (1990), 1-21. 
3. Driankov, D., Hellendoorn, H. and Reinfrank, M., “An Introduction to Fuzzy Control," (Springer-Verlag, 1993).

4. Garnell, P., East, D.J., “Guided Weapon Control Systems” (Pergamon Press, 1997).

5. Kitamura, S. and T. Kurozumi, "Extended Circle Criterion and Stability Analysis of Fuzzy Control Systems," Proc. of the Int. Fuzzy Engineering Symp. IFES'91 (Nov. 13-15, 1991, Jokohama, Japan), vol. 2: Fuzzy Engineering toward Human Friendly Systems, 634-643.

6. Koprinkova, P., "Membership Functions Shape Influence on Fuzzy Control Actions," The First Workshop on Fuzzy Based Expert Systems FUBEST'94 (Sept. 28-30, 1994, Sofia, Bulgaria), 116-118.

7. Koprinkova, P., "Influence Investigation of the Shape of Fuzzy Variables Membership Functions on the Control Surface Shape of a Fuzzy Logic Controller," in Proceedings of the National Conference 'Automatics and Informatics'95,' (Nov. 7-9, 1995, Sofia, Bulgaria), 22-25 (in Bulgarian).

8. Koprinkova, P., "Membership Functions Shape and Its Influence on the Dynamical Behaviour of Fuzzy Logic Controller," Cybernetics and Systems: An International Journal 31, 2 (March 2000), 161-173.

9. Koprinkova, P., "Membership Functions Shape and Its Influence on the Stability of Fuzzy Control Systems," Cybernetics and Systems: An International Journal (in press).

10. Lee, C.-C., "Fuzzy logic in Control Systems: Fuzzy Logic Controller - Part I and Part II," IEEE Trans. on SMC 20 (1990), 404-434.

PETYA KOPRINKOVA is a research assistant at the Institute of Control and System Research, Bulgarian Academy of Sciences. She holds an M.Sc. degree from the Technical University of Sofia. Ms Koprinkova has more than twenty publications in the areas of control of biotechnical systems, fuzzy logic and neural networks. Address: Acad. G. Bonchev Str., block 2, P.O.Box 79, Sofia 1113, BULGARIA. 